Introduction Disseminated neonatal herpes is one of the deadliest perinatal infections with a reported mortality of $85 \%$ in the untreated neonate with early commencement of aciclovir reducing this to $30 \%$. Timely recognition is often difficult because of the non- specific presentation which delays the initiation of aciclovir.

Case Series Report We report 3 cases of severe disseminated neonatal herpes (virology positive) seen in a year at our hospital with varying presentations and outcomes.

Case 1: A baby (born at $36+6$ weeks by caesarean section) presented on day 9 with lethargy and reduced feeding whereupon a septic screen was completed with commencement of intravenous antibiotics. On Day 10, the baby developed severe hyponatremia resistant to treatment and was therefore referred to a tertiary hospital at day 12 where he developed liver failure and disseminated intravascular coagulation (DIC). Despite aggressive management the baby died at day 25. Aciclovir was only started at day 14 .

Case 2: A $36+1$ week baby born by caesarean section developed respiratory distress at day4 of life with rapid deterioration and chest X-ray changes. The baby was ventilated and transferred to a tertiary centre where he developed severe shock, liver failure and DIC. Aciclovir was started on day 5 but the baby succumbed and died on day 6 .

Case 3: A term baby born by normal vaginal delivery was admitted on day 9 with 2 day history of poor feeding, breathing difficulties and ulcer on the tongue. He was commenced on IV antibiotics and aciclovir. The bloods showed evidence of DIC and liver failure. The baby was transferred to a liver unit where he received intensive treatment for more than 1 month with gradual recovery following discharge.

Discussion Disseminated neonatal herpes presents in varying scenarios often with symptoms starting between day 4-9. Of the three cases only one was commenced on aciclovir immediately on admission who eventually survived. Early commencement of aciclovir is crucial in neonates presenting with poor feeding, vesicular rash, severe hyponatremia, oral ulcers, seizures, bleeding etc. after 4 days of life.

\section{G593(P) CASE REPORT: AN UNUSUAL PRESENTATION OF RHEUMATIC FEVER IN A 3 YEAR OLD CHILD IN THE UK}

${ }^{1}$ TM Miller, ${ }^{2} \mathrm{DB}$ Blundell, ${ }^{1} \mathrm{YA}$ Aung, ${ }^{1} \mathrm{AK}$ Kona, ${ }^{3,4} \mathrm{ME}$ Emonts. ${ }^{1}$ Children and Young Peoples Department, West Cumberland Hospital, North Cumbria University Hospital Trust, Carlisle, UK; ${ }^{2}$ Paediatric Cardiology Department, Freeman Hospital, Newcastle upon Tyne Hospitals NHS FT, Newcastle upon Tyne, UK; ${ }^{3}$ Department of Paediatric Immunology, Infectious Diseases and Allergy, Great North Children's Hospital, Newcastle upon Tyne Hospitals NHS FT, Newcastle upon Tyne, UK; ${ }^{4}$ Institute of Cellular Medicine, Newcastle University, Newcastle upon Tyne, UK

\subsection{6/archdischild-2020-rcpch.510}

We describe an unusual presentation of a 3-year-old to a UK District General Hospital with symptomatology initially thought to represent a viral illness, however, was subsequently diagnosed with acute rheumatic fever. The patient had a 6week evolving presentation favouring the diagnosis which included a rash, followed by an elevated streptolysin titre and arthralgia, then finally a fever with an associated pan-systolic murmur. The difficulty with diagnosis was that these symptoms were occurring in a young patient over an extended period of time and acute rheumatic fever is rare in toddlers.

The young child was transferred to the local tertiary centre for further management; however, she went on to develop acute left ventricular failure. Rheumatic fever can lead to rheumatic heart disease which can have long term health outcomes for patients such as demonstrated in this case report.

The district general hospital and the tertiary centre reviewed practice and discussed discrepancies around the diagnosis of this case. Following transfer to the tertiary centre, the team recalled the history and identified images that the parent had documented of a typical erythema marginatum rash; this was not visualised at the time of admission. This raised an interesting point around the power of digital imaging in today's practice for aiding diagnosis. Throughout the course of the condition the child's parent continued to raise concerns which was invaluable in obtaining the right diagnosis for the child.

\section{Take-home messages}

- Always be willing to re-evaluate diagnosis and listen to parental concerns

- This case illustrates the need to be vigilant for the presentation of rare illness as they can have a significant impact on the quality of life of young patients

- Digital imaging can play a vital role in diagnosis of serious diseases

\section{G594(P) A CASE OF SUSPECTED GRISCELLI SYNDROME}

${ }^{1}$ AK Ndululu, ${ }^{2}$ A Migowa. Paediatrics, Aga Khan University Hospital, Nairobi, Kenya

\subsection{6/archdischild-2020-rcpch.511}

Aims Primary immunodeficiencies are not often documented, but the prevalence in developed countries is estimated at approximately 1 in 1200. Lack of statistics on the SubSaharan African population leads to inappropriate management, poor quality of life and markedly reduced survival. This presentation aims to highlight the need for timely identification and early diagnosis of primary immunodeficiencies, hence improved care and survival of affected patients.

Methods A retrospective chart review of a patient who presented with a history of recurrent respiratory tract infections since the age of four months, often requiring inpatient care two to three times every year.

Results The history included infantile eczema and recurrent respiratory infections for which the patient had been on follow up at the pediatric pulmonology clinic. She also had global developmental delay and was being followed up at the pediatric neurology clinic. There was a history of widespread skin abscesses which resolved on treatment. At 6 years, she was non- verbal, could only walk short distances and was on home oxygen.

Examination revealed a dull looking, wasted child who was pale and had sparse, brittle, silvery hair, poor dentition, global hypotonia, reduced muscle bulk, and skin depigmentation with features of longstanding eczema. Weight for age was at the $3 \mathrm{rd}$ centile. She was saturating at $75 \%$ in room air and a chest CT scan revealed lower lung lobe collapse with a possible diagnosis of interstitial lung disease. Abdominal examination showed hepatomegaly. There were no murmurs on auscultation, but there was severe pulmonary arterial hypertension on echocardiography.

Given the frequent infections, skin depigmentation, silvery hair and global developmental delay, a diagnosis of suspected Griscelli syndrome was made. The patient passed away at the age of 6 years while on home care. 